\title{
ON SPECIAL DEVELOPABLE RULED SURFACES WITH POINTWISE 1-TYPE GAUSS MAP
}

\author{
O. KAYA AND M. ÖNDER
}

Received 10 October, 2020

\begin{abstract}
In this paper, some special developable ruled surfaces with point-wise Gauss map are studied. Three different special developable ruled surfaces called rectifying ruled surface, generalized normal ruled surface and osculating type ruled surface are considered. The conditions for such surfaces to have 1-type Gauss map and also to be minimal are introduced. Finally, some examples are given for the obtained results.
\end{abstract}

2010 Mathematics Subject Classification: 53A25; 53B25; 53C40

Keywords: ruled surface, Laplacian, Gauss map, minimal surface

\section{INTRODUCTION}

Studies on finite type submanifolds in the Euclidean and semi-Euclidean spaces have started in 1970's by Chen and then this subject has been used for characterizing and investigating other important submanifolds $[4,5]$. After this subject has been introduced, it has gained interest among geometers and developed rapidly. Especially, the application of the notion of finite type to Gauss map of submanifolds has been an important development $[1-3,6,7,13,14,16]$. The notion of 1-type Gauss map is described by the equality of $\Delta G=\lambda(G+C)$ where $\Delta$ is the Laplacian of a surface $M$, $G$ is the Gauss map of $M, \lambda$ is a real number and $C$ is a constant vector [13]. Some of the well-known surfaces with 1-type Gauss map in the Euclidean 3-space $E^{3}$ are catenoids, helicoids and right cones [6].

In the current paper, our purpose is to study some special ruled surfaces that have pointwise 1-type Gauss map whose details are given in Section 2. These surfaces are called rectifying, generalized normal and osculating type ruled surfaces defined in $[11,12,15]$. We obtain characterizations for such surfaces to have pointwise 1-type Gauss map. We also give results when such surfaces are minimal.

\section{PRELIMINARIES}

An isometric immersion (a smooth map, respectively) $x: M \rightarrow E^{3}$ of a submanifold (a 2-dimensional Riemannian manifold, respectively) $M$ in the Euclidean 3-space 
$E^{3}$ is of finite type if the position vector field $x$ of the submanifold $M$ can be given as a finite sum of the eigenvectors of Laplacian $\Delta$ of $M$ which is $x=x_{0}+\sum_{i=1}^{j} x_{i}$ where $x_{0}$ is a constant map and $x_{1}, x_{2}, \ldots, x_{j}$ are non-constant maps such that $\Delta x_{i}=\lambda_{i} x_{i}$, $\lambda_{i} \in \mathbb{R}, 1 \leq i \leq j$. If the eigenvalues $\lambda_{1}, \lambda_{2}, \ldots \lambda_{j}$ are different from each other, then $M$ is of $j$-type. Especially, if one of the eigenvalues $\lambda_{i}$ vanishes, then $M$ is of null $j$-type $[4,5]$.

Let $M$ be a connected (not necessarily compact) surface in the Euclidean 3-space $E^{3}$. Then the position vector field $x$ and the mean curvature vector $\mathrm{H}$ of the surface $M$ in the Euclidean 3-space $E^{3}$ satisfy the equation $\Delta x=-2 \mathrm{H}$ where $\mathrm{H}=H G, H$ is the mean curvature and $G$ is the Gauss map of the surface. This equality leads to a well-recognized result which is: A surface $M$ in the Euclidean 3-space $E^{3}$ is a minimal surface if and only if all coordinate functions of $E^{3}$ that are restricted to the surface $M$ are harmonic functions, i.e.,

$$
\Delta x=0
$$

$[4,16]$.

In a Euclidean space, a submanifold $M$ is called to be of 1-type, i.e., the position vector field $x$ of $M$ satisfies the equation $\Delta x=\lambda x, \lambda \in \mathbb{R}$ if and only if either the submanifold minimal $(\lambda=0)$ or $M$ is a minimal submanifold of a hypersphere centered at the origin $(\lambda \neq 0)$ of the Euclidean space which $M$ is in [16].

Let $M$ be a surface in $E^{3}$ with the parametric form $x=x(u, v)$. The Gauss map (or the unit surface normal) of $M$ is defined by

$$
G: M \rightarrow S^{2} \subset E^{3}, G(u, v)=\left(\frac{x_{u} \times x_{v}}{\left\|x_{u} \times x_{v}\right\|}\right)(u, v)
$$

where $S^{2}$ denotes the unit sphere and $x_{u}=\partial x / \partial u, x_{v}=\partial x / \partial v$ are partial derivatives [8].

Let the matrix $\left(g_{i j}\right)$ consist of the components of the Riemannian metric on $M$. Then,

$$
\left(g_{i j}\right)=\left[\begin{array}{ll}
g_{11} & g_{12} \\
g_{21} & g_{22}
\end{array}\right]=\left[\begin{array}{ll}
\left\langle x_{u}, x_{u}\right\rangle & \left\langle x_{u}, x_{v}\right\rangle \\
\left\langle x_{v}, x_{u}\right\rangle & \left\langle x_{v}, x_{v}\right\rangle
\end{array}\right] .
$$

If the matrix $\left(g^{i j}\right)$ is the inverse of $\left(g_{i j}\right)$ and $g$ is the determinant of $\left(g_{i j}\right)$, then, the Laplacian $\Delta$ on $M$ is given by

$$
\Delta=-\frac{1}{\sqrt{g}} \sum_{i, j} \frac{\partial}{\partial x_{i}}\left(\sqrt{g} g^{i j} \frac{\partial}{\partial x_{j}}\right) .
$$

The surface $M$ has pointwise 1-type Gauss map if the Gauss map $G$ satisfy the condition

$$
\Delta G=f(G+C)
$$

where $f=f(u, v)$ is a real valued function and $C$ is a constant vector. A pointwise 1-type Gauss map is called proper if the function $f$ given in (2.3) is non-constant. 
If the vector $C$ vanishes, then the surface is called a surface with pointwise 1-type Gauss map of the first kind. If not, it is called of the second kind [6].

Here, we give some examples of surfaces with pointwise 1-type Gauss map.

Example 1. Let the surface $M_{1}$ be given by the parametrization $x(u, v)=(u, 0,0)+$ $v(0, \cos u, \sin u)$, whose Gauss map is $G(u, v)=\frac{1}{\sqrt{v^{2}+1}}(-v,-\sin u, \cos u)$. Then, we get $\Delta G=\frac{2}{\left(v^{2}+1\right)^{2}} G$ where $f=\frac{2}{\left(v^{2}+1\right)^{2}}$ and $C=0$. Therefore, the surface $M_{1}$ has pointwise 1-type Gauss map of the first kind. On the other hand, the Laplacian of the position vector $x$ of the surface $M_{1}$ vanishes, i.e., $\Delta x=0$. Then, $M_{1}$ is minimal. The graph of the surface $M_{1}$ is given in Figure 1.
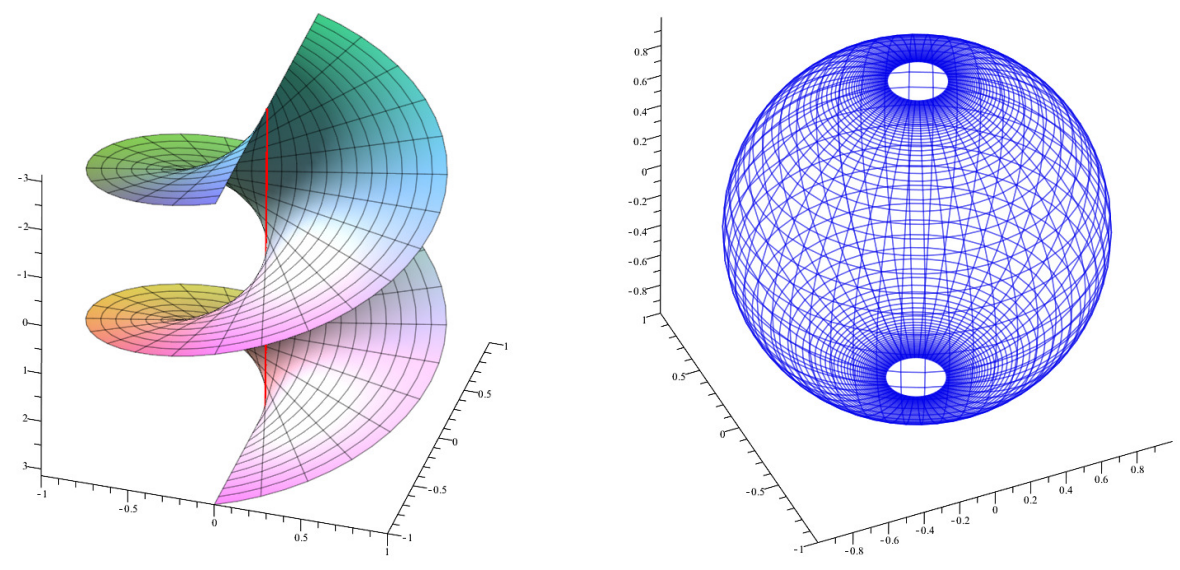

FIGURE 1. $M_{1}$ with its base curve $\alpha$ (red) and Gauss map (blue).

Example 2. Let the surface $M_{2}$ be given by the parametrization $x(u, v)=(v \cos u$, $v \sin u, v)$, whose Gauss map is $G(u, v)= \pm \frac{\sqrt{2}}{2}(\cos u, \sin u,-1)$. Then, we obtain $\Delta G=v^{2}\left[G \pm\left(0,0, \frac{\sqrt{2}}{2}\right)\right]$ where $f=v^{2}$ and $C= \pm\left(0,0, \frac{\sqrt{2}}{2}\right)$. Therefore, the surface $M_{2}$ has pointwise 1-type Gauss map of second kind. The surface $M_{2}$ does not have harmonic type position vector, thus it is not minimal. The graph of the surface $M_{2}$ is given in Figure 2.

Let $\alpha: I \subset \mathbb{R} \rightarrow E^{3}$ be a regular curve in the Euclidean 3-space $E^{3}$ given by the arc length parameter $u$ and with Frenet frame $\{T, N, B\}$, curvature $\kappa$ and torsion $\tau$. The vector fields $T, N, B$ defined by $T=\alpha^{\prime}, N=\alpha^{\prime \prime} /\left\|\alpha^{\prime \prime}\right\|, B=T \times N$ are called unit tangent, principal normal and binormal vector, respectively, where the prime denotes the derivative with respect to $u$. Then, $\{T, N, B\}$ is an orthogonal moving frame along $\alpha$ and called Frenet frame of it. The derivative formulae (or Frenet formulae) of that frame are given by $T^{\prime}=\kappa N, N^{\prime}=-\kappa T+\tau B, B^{\prime}=-\tau N$. The 

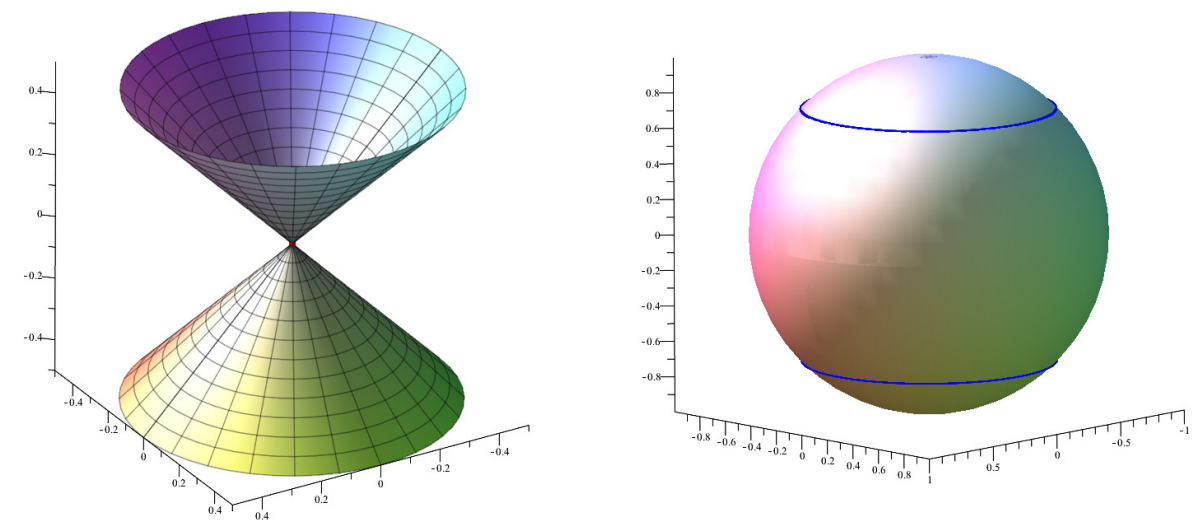

FIGURE 2. $M_{2}$ with its base curve $\alpha$ (red) and Gauss map (blue).

planes $S p\{T, N\}, S p\{T, B\}$ and $S p\{N, B\}$ are called the osculating, the rectifying and the normal planes of $\alpha$, respectively [8].

A surface $M: I \times J \rightarrow \mathbb{R}^{3}, I, J \subset \mathbb{R}$ given by the parametrization $x(u, v)=\alpha(u)+$ $v q(u)$ is called a ruled surface where $u \in I$ and $v \in J$. The curve $\alpha$ is called base curve and $q$ is called director curve. The straight lines $v \rightarrow \alpha(u)+v q(u)$ are called rulings [10]. Clearly, a ruled surface is constructed by the continuous motion of a straight line and that makes this surface important in some areas such as computer aided geometric design and architecture [9]. A ruled surface is called developable if and only if $\operatorname{det}\left(\alpha^{\prime}, q, q^{\prime}\right)=0$ [10].

\section{DEVELOPABLE GENERAL RECTIFYING RULED SURFACES WITH POINTWISE 1-TYPE GAUSS MAP}

The parametric form of a general rectifying ruled surface $M$ is given by $x(u, v)=$ $\alpha(u)+v q_{r}(u), \quad q_{r}(u)=a_{1}(u) T(u)+a_{2}(u) B(u)$ where $u$ is the arc-length parameter of $\alpha, a_{1}=a_{1}(u), a_{2}=a_{2}(u)$ are smooth functions of $u$ such that $a_{1}^{2}+a_{2}^{2}=1$ and the index " $r$ " is used to remark that the ruling always lies on the general rectifying plane of the base curve $\alpha$ [15]. The general rectifying ruled surface $M$ is developable if and only if $a_{1} \kappa-a_{2} \tau=0$ [15]. Hereafter, we will assume that the general rectifying ruled surface $M$ is developable.

The Gauss map $G$ of the developable general rectifying surface (dgr-surface) $M$ is $G(u, v)=-N(u)$. Then, we obtain

$$
G_{u}=\kappa T-\tau B, G_{u u}=\kappa^{\prime} T+\left(\kappa^{2}+\tau^{2}\right) N-\tau^{\prime} B, G_{v}=G_{u v}=G_{v u}=G_{v v}=0 .
$$

Also, the components and the determinant $g$ of the matrix $\left(g_{i j}\right)$ are obtained as $g_{11}=\left(1+v a_{1}^{\prime}\right)^{2}+v^{2}\left(a_{2}^{\prime}\right)^{2}, g_{12}=g_{21}=a_{1}, g_{22}=1$ and $g=\left(1+v a_{1}^{\prime}\right)^{2}+v^{2}\left(a_{2}^{\prime}\right)^{2}-a_{1}^{2}$ 
respectively. From (2.2), the Laplacian operator on the $d g r$-surface $M$ is

$$
\Delta=-\xi\left[\frac{\partial}{\partial u}\left(\xi \frac{\partial}{\partial u}-a_{1} \xi \frac{\partial}{\partial v}\right)+\frac{\partial}{\partial v}\left(-a_{1} \xi \frac{\partial}{\partial u}+\xi\left[\left(1+v a_{1}^{\prime}\right)^{2}+v^{2}\left(a_{2}^{\prime}\right)^{2}\right] \frac{\partial}{\partial v}\right)\right]
$$

where $\xi$ is a smooth function given by $\xi(u, v)=\frac{1}{\sqrt{\left(1+v a_{1}^{\prime}\right)^{2}+v^{2}\left(a_{2}^{\prime}\right)^{2}-a_{1}^{2}}}$. Then, the Laplacian of the Gauss map of $M$ is computed as

$$
\Delta G=-\xi\left(\kappa\left(\xi_{u}-a_{1} \xi_{v}\right)+\kappa^{\prime} \xi\right) T-\left(\kappa^{2}+\tau^{2}\right) \xi^{2} N+\xi\left(\tau\left(\xi_{u}-a_{1} \xi_{v}\right)+\tau^{\prime} \xi\right) B .
$$

From (3.1) we have that $\Delta G=0$ if and only if $\kappa=0, \tau=0$. Then, the following theorem is obtained:

Theorem 1. A dgr-surface $M$ has harmonic type Gauss map $G$ if and only if its base curve $\alpha$ is a straight line.

Moreover, from (3.1), it yields that $\Delta G=f G, f=\left(\kappa^{2}+\tau^{2}\right) \xi^{2}$ if and only if the equations $\kappa\left(\xi_{u}-a_{1} \xi_{v}\right)+\kappa^{\prime} \xi=0, \tau\left(\xi_{u}-a_{1} \xi_{v}\right)+\tau^{\prime} \xi=0$ hold and we have the following theorem:

Theorem 2. A dgr-surface $M$ has point-wise 1-type Gauss map of the first kind if and only if the curvatures do not vanish at the same time and the system

$$
\kappa\left(\xi_{u}-a_{1} \xi_{v}\right)+\kappa^{\prime} \xi=0, \quad \tau\left(\xi_{u}-a_{1} \xi_{v}\right)+\tau^{\prime} \xi=0
$$

holds.

Theorem 2 gives us the following special cases:

Case 1: Let the base curve $\alpha$ be a plane curve different from a straight line. Then, $\kappa \neq 0, \tau=0$. From the developable condition we have $a_{1}=0, a_{2}=1, \xi=1$ and (3.1) becomes $\Delta G=-\left(\kappa^{\prime} T+\kappa^{2} N\right)$. We have that $\Delta G=f G$ with $f=\kappa^{2}$ if and only if $\kappa$ is constant. Then, we obtain the following theorem:

Theorem 3. Let the base curve $\alpha$ be a plane curve. Then the dgr-surface $M$ has the pointwise 1-type Gauss map of the first kind if and only if $\alpha$ is a circle.

Case 2: Let the base curve $\alpha$ be a circular helix with non-zero curvatures $\kappa, \tau$. Then, from the system (3.2) we have that $\xi_{u}-a_{1} \xi_{v}=0$ which gives the following theorem:

Theorem 4. Let the base curve $\alpha$ be a circular helix. Then the dgr-surface $M$ has the pointwise 1-type Gauss map of the first kind if and only if $\xi_{u}-a_{1} \xi_{v}=0$ holds.

Case 3: Assume that one of the curvatures $\kappa, \tau$ is non-zero constant. Then, from the system (3.1), we have that the other one is also non-zero constant and following theorem is obtained:

Theorem 5. If one of the curvature functions $\kappa, \tau$ of base curve $\alpha$ is a non-zero constant, then the dgr-surface $M$ has the pointwise 1-type Gauss map of the first kind if and only if $\alpha$ is a circular helix. 
Now let us examine the Laplacian of the position vector $x$ of $d g r$-surface $M$. For any surface $M$ we have that $\Delta x=0$ holds if and only if $M$ is minimal [16]. Also, we know that the $d g r$-surface $M$ is minimal if and only if $\left(1+v a_{1}^{\prime}\right) \kappa-v a_{2}^{\prime} \tau=0$ holds [15]. Then, by taking the partial derivatives of $x$, from (2.2) it follows

$$
\begin{aligned}
\Delta x=- & \xi\left\{\left(1+v a_{1}^{\prime}\right) \xi_{u}+\left(v a_{1}^{\prime \prime}-2 a_{1} a_{1}^{\prime}\right) \xi-a_{1}\left(a_{1} \xi\right)_{u}\right. \\
& \left.-\left(1+v a_{1}^{\prime}\right) a_{1} \xi_{v}+a_{1}\left[\left(\left(1+v a_{1}^{\prime}\right)^{2}+v^{2}\left(a_{2}^{\prime}\right)^{2}\right) \xi\right]_{v}\right\} T \\
- & \xi^{2}\left\{\left(1+v a_{1}^{\prime}\right) \kappa-v a_{2}{ }_{2} \tau\right\} N \\
- & \xi\left\{v a^{\prime}{ }_{2} \xi_{u}+\left(v a^{\prime \prime}{ }_{2}-2 a_{1} a^{\prime}{ }_{2}\right) \xi-a_{2}\left(a_{1} \xi\right)_{u}\right. \\
& \left.-v a_{1} a_{2}^{\prime} \xi_{v}+a_{2}\left[\left(\left(1+v a_{1}^{\prime}\right)^{2}+v^{2}\left(a_{2}^{\prime}\right)^{2}\right) \xi\right]_{v}\right\} B .
\end{aligned}
$$

We have that $\Delta x=0$ if and only if the system

$$
\left\{\begin{array}{l}
\left(1+v a_{1}^{\prime}\right)\left(\xi_{u}-a_{1} \xi_{v}\right)+\left(v a^{\prime \prime}{ }_{1}-2 a_{1} a_{1}\right) \xi \\
\quad-a_{1}\left(a_{1} \xi\right)_{u}+a_{1}\left[\left(\left(1+v a_{1}^{\prime}\right)^{2}+v^{2}\left(a_{2}^{\prime}\right)^{2}\right) \xi\right]_{v}=0 \\
\left(1+v a_{1}^{\prime}\right) \kappa-v a_{2}^{\prime} \tau=0 \\
v a_{2}^{\prime}{ }_{2}\left(\xi_{u}-a_{1} \xi_{v}\right)+\left(v a^{\prime \prime}{ }_{2}-2 a_{1} a_{2}^{\prime}\right) \xi \\
\quad-a_{2}\left(a_{1} \xi\right)_{u}+a_{2}\left[\left(\left(1+v a_{1}\right)^{2}+v^{2}\left(a_{2}^{\prime}\right)^{2}\right) \xi\right]_{v}=0
\end{array}\right.
$$

holds. Then, we get the followings:

Theorem 6. Let $M$ be a dgr-surface. Then, the followings are equivalent:

(i) M has harmonic type position vector.

(ii) $M$ is minimal.

(iii) $\left(1+v a_{1}^{\prime}\right) \kappa-v a_{2}^{\prime} \tau=0$ holds.

(iv) The system in (3.4) holds.

Using the equality $\left(1+v a_{1}^{\prime}\right) \kappa-v a_{2}^{\prime} \tau=0$, the first and third equalities in (3.4) become

$$
\begin{gathered}
v a_{2}^{\prime} \frac{\tau}{\kappa}\left(\xi_{u}-a_{1} \xi_{v}\right)+\left(v a_{1}^{\prime \prime}-2 a_{1} a_{1}^{\prime}\right) \xi-a_{1}\left(a_{1} \xi\right)_{u}+a_{1}\left[\left(v^{2}\left(a_{2}^{\prime}\right)^{2}\left(1+\frac{\tau^{2}}{\kappa^{2}}\right)\right) \xi\right]_{v}=0 \\
v a_{2}^{\prime}\left(\xi_{u}-a_{1} \xi_{v}\right)+\left(v a_{2}^{\prime \prime}-2 a_{1} a_{2}^{\prime}\right) \xi-a_{2}\left(a_{1} \xi\right)_{u}+a_{2}\left[\left(v^{2}\left(a_{2}^{\prime}\right)^{2}\left(1+\frac{\tau^{2}}{\kappa^{2}}\right)\right) \xi\right]_{v}=0
\end{gathered}
$$

respectively. Then, we get

$$
v a_{2}^{\prime}\left(\xi_{u}-a_{1} \xi_{v}\right)\left(a_{2} \frac{\tau}{\kappa}-a_{1}\right)+a_{2}\left(v a_{1}^{\prime \prime}-2 a_{1} a_{1}^{\prime}\right) \xi-a_{1}\left(v a_{2}^{\prime \prime}-2 a_{1} a_{2}^{\prime}\right) \xi=0 .
$$


Since the surface is developable, we have $a_{1} \kappa-a_{2} \tau=0$ which gives $a_{2}\left(v a_{1}^{\prime \prime}-2 a_{1} a_{1}^{\prime}\right)-$ $a_{1}\left(v a_{2}^{\prime \prime}-2 a_{1} a_{2}^{\prime}\right)=0$ and following theorem is given:

Theorem 7. If the dgr-surface $M$ has the harmonic type position vector (or, equivalently minimal), then

holds.

$$
\frac{\tau}{\kappa}=\frac{v a_{1}^{\prime \prime}-2 a_{1} a_{1}^{\prime}}{v a_{2}^{\prime \prime}-2 a_{1} a_{2}^{\prime}}
$$

Example 3. Let the space curve $\alpha$ be given by the parametrization $\alpha(u)=\left(\cos \frac{u}{\sqrt{2}}\right.$, $\left.\sin \frac{u}{\sqrt{2}}, \frac{u}{\sqrt{2}}\right)$. Then, by calculating its Frenet elements and choosing $a_{1}(u)=a_{2}(u)=$ $1 / \sqrt{2}$, we obtain the $d g r$-surface $M_{3}$ with the parametrization $x(u, v)=\left(\cos \frac{u}{\sqrt{2}}\right.$, $\left.\sin \frac{u}{\sqrt{2}}, \frac{u}{\sqrt{2}}+v\right)$. The Gauss map of $d g r$-surface $M_{3}$ and the Laplacian of Gauss map are computed as $G(u, v)=\left(\cos \frac{u}{\sqrt{2}}, \sin \frac{u}{\sqrt{2}}, 0\right)$ and $\Delta G=\left(\cos \frac{u}{\sqrt{2}}, \sin \frac{u}{\sqrt{2}}, 0\right)$, respectively. Finally, we obtain that $\Delta G=G$ which indicates that $M_{3}$ has 1-type Gauss map of first kind. This result satisfies Theorem 2. The second equation given in Theorem 6 is not satisfied since $\left(1+v a_{1}^{\prime}\right) \kappa-v a_{2}^{\prime} \tau=\frac{1}{2} \neq 0$. Then, the $d g r$-surface $M_{3}$ is not minimal. So, it does not have harmonic type position vector. The graph of the $d g r$-surface $M_{3}$ is given in Figure 3.
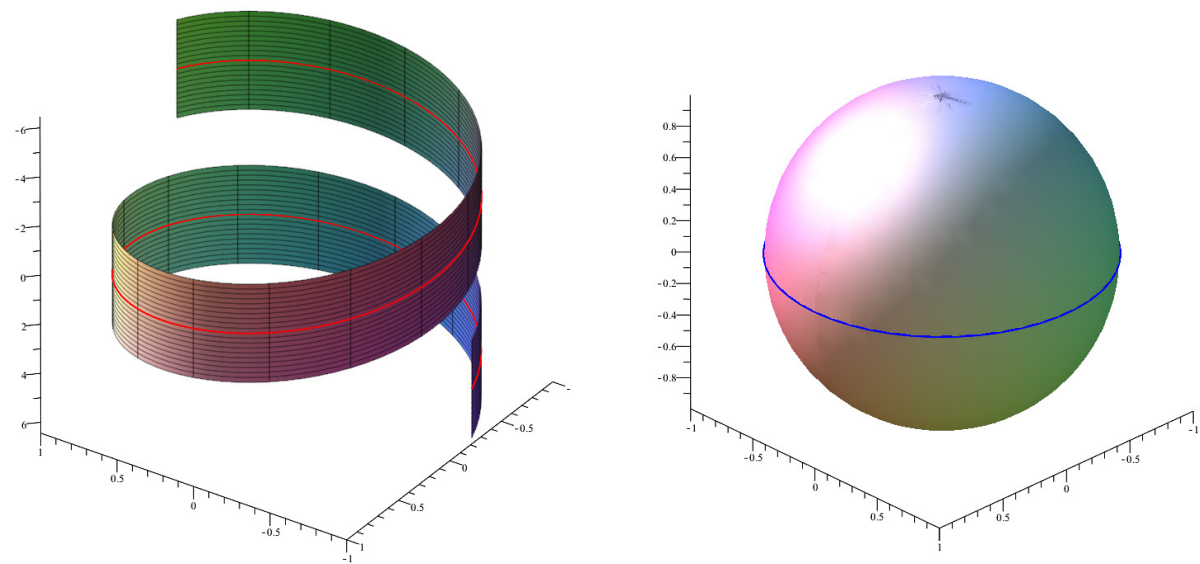

FIGURE 3. $M_{3}$ with its base curve $\alpha$ (red) and Gauss map (blue).

\section{DEVELOPABLE GENERALIZED NORMAL RULED SURFACES WITH POINTWISE 1-TYPE GAUSS MAP}

The parametric form of a generalized normal surface $M$ is given by $x(u, v)=$ $\alpha(u)+v q_{n}(u), \quad q_{n}(u)=b_{1}(u) T(u)+b_{2}(u) B(u)$ where $u$ is the arc length parameter 
of base curve $\alpha, b_{1}=b_{1}(u), b_{2}=b_{2}(u)$ are smooth functions of $u$ such that $b_{1}^{2}+b_{2}^{2}=$ 1 and the index " $n$ " is used to remark that the ruling always lies on the normal plane of the base curve $\alpha$ [12]. Such a surface is developable if and only if $b_{1}^{\prime} b_{2}-b_{1} b_{2}^{\prime}-\tau=0$ holds. For the developable generalized normal surface ( $d g n$-surface) $M$, we also have $b_{1} \tau+b_{2}^{\prime}=0, b_{1}^{\prime}-b_{2} \tau=0$ [12].

The Gauss map $G$ of the dgn-surface $M$ is obtained as $G(u, v)=-b_{2}(u) N(u)+$ $b_{1}(u) B(u)$. The matrix $\left(g_{i j}\right)$ has the form

$$
\left(g_{i j}\right)=\left(\begin{array}{cc}
\left(1-v b_{1} \kappa\right)^{2} & 0 \\
0 & 1
\end{array}\right) .
$$

The determinant $g$ is computed as $g=\left(1-v b_{1} \kappa\right)^{2}$. Finally, from (2.2) the Laplacian operator on $d g n$-surface $M$ is

$$
\Delta=-\frac{1}{\psi}\left[\frac{\partial}{\partial u}\left(\frac{1}{\psi} \frac{\partial}{\partial u}\right)+\frac{\partial^{2}}{\partial v^{2}}\right]
$$

where $\psi$ is a differentiable function defined by $\psi(u, v)=1-v b_{1} \kappa$. Then, from (4.1) the Laplacian of the Gauss map is

$$
\Delta G=\frac{\left[b_{2} \kappa \psi\right]_{u}}{\psi^{3}} T-\frac{b_{2} \kappa^{2}}{\psi^{2}} N
$$

and we have the followings:

Theorem 8. A dgn-surface $M$ has pointwise 1-type Gauss map of the first kind, that is $\Delta G=f G$ where $f=\kappa^{2}$ if and only if $\kappa=$ constant and $b_{1}=0$.

Proof. From (4.2), we obtain that $\Delta G=f G$ holds if and only if $\left[b_{2} \kappa \psi\right]_{u}=0$ and $b_{1}=0$ which lead us to the desired result.

Now, let us examine the Laplacian of the position vector $x$ of the dgn-surface $M$. From (4.1), we obtain

$$
\Delta x=\frac{b_{2} \kappa}{\psi}\left(-b_{2} N+b_{1} B\right) .
$$

Then, from the equality $\Delta x=0$, we get the following:

Theorem 9. A dgn-surface $M$ has harmonic type position vector (or, equivalently minimal) if and only if $b_{2}=0$ or $\kappa=0$, i.e., $\alpha$ is a straight line.

Moreover, from (4.3), we also have the following theorem:

Theorem 10. The position vector $x$ and Gauss map $G$ of a dgn-surface M satisfy the equation $\Delta x=\frac{b_{2} \kappa}{\psi} G$.

Using the equality $\Delta x=-2 \mathrm{H}$ given in [4], from Theorem 10 we have that the mean curvature $H$ of a $d g n$-surface $M$ is $H=-\frac{b_{2} \kappa}{2 \psi}$, where $\mathrm{H}=H G$ is the mean curvature vector and $H$ is the mean curvature of $M$. This result also coincides with the calculated value of mean curvature $H$ of a $d g n$-surface $M$ in [12]. 
Example 4. Let choose the unit circle $\alpha(u)=(\cos u, \sin u, 0)$ as the base curve. By taking, $b_{1}=0, b_{2}=1$, the parametric form of a dgn-surface $M_{4}$ is obtained as $x(u, v)=(\cos u, \sin u, v)$. The Gauss map of $M_{4}$ is $G=(\cos u, \sin u, 0)$. Then, by a simple calculation, we obtain $\Delta G=G=(\cos u, \sin u, 0)$ which shows that the $d g n$ surface $M_{4}$ has 1-type Gauss map. Since $b_{2} \neq 0$ or $\kappa \neq 0$, by Theorem 9, the $d g n$ surface $M_{4}$ is not minimal. Then, it does not have harmonic type position vector. The graph of the $d g n$-surface $M_{4}$ is given in Figure 4.
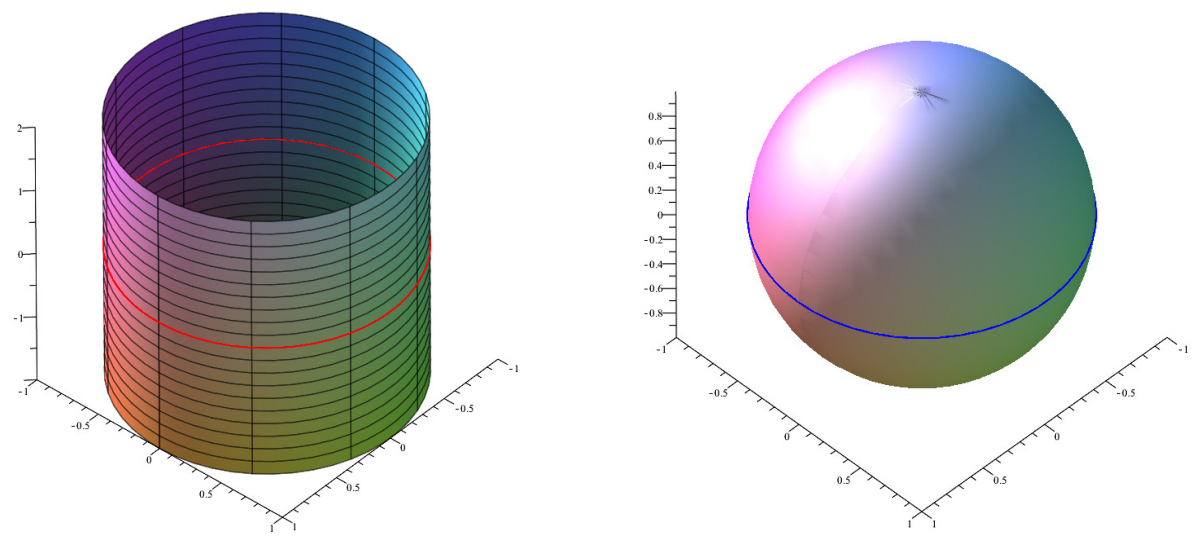

FIGURE 4. $M_{4}$ with its base curve $\alpha$ (red) and Gauss map (blue).

\section{Developable osculating type RUled surfaces With 1-TyPe Gauss}

MAP

An osculating type ruled surface $M$ has the parametric form $x(u, v)=\alpha(u)+$ $v q_{o}(u), q_{o}(u)=\cos \theta T(u)+\sin \theta N(u)$ where $u$ is the arclength parameter of base curve $\alpha, \theta=\theta(u)$ is a smooth function of $u$ and the index " $o$ " is used to remark that the ruling always lies on the osculating plane of the base curve $\alpha$ [11]. A new orthonormal frame $\left\{q_{o}, B, r\right\}$ has been defined for osculating type ruled surfaces where $r=q_{o} \times B$. The derivative formulae for this frame are

$$
\left[\begin{array}{c}
q_{o}^{\prime} \\
B^{\prime} \\
r^{\prime}
\end{array}\right]=\left[\begin{array}{ccc}
0 & \mu & -\eta \\
-\mu & 0 & \omega \\
\eta & -\omega & 0
\end{array}\right]\left[\begin{array}{c}
q_{o} \\
B \\
r
\end{array}\right]
$$

where $\mu=\tau \sin \theta, \omega=\tau \cos \theta, \eta=\theta^{\prime}+\kappa$ [11]. The osculating type ruled surface $M$ is developable if and only if $\tau=0$ or $\sin \theta=0$. In both cases, we get $\mu=0$. 
From (2.2), the Laplacian operator on developable osculating type ruled surface (dot-surface) $M$ is obtained as

$$
\Delta=-\zeta\left[\frac{\partial}{\partial u}\left(\zeta^{3} \frac{\partial}{\partial u}-\zeta^{3} \cos \theta \frac{\partial}{\partial v}\right)+\frac{\partial}{\partial v}\left(-\zeta^{3} \cos \theta \frac{\partial}{\partial u}+\zeta^{3}\left[\zeta^{-2}+\cos ^{2} \theta\right] \frac{\partial}{\partial v}\right)\right]
$$

where $\zeta(u, v)=\frac{1}{\sin \theta-v \eta}$ is a smooth function. The Gauss map of the dot-surface $M$ is obtained as $G(u, v)=B(u)$. In order to compute the Laplacian of the Gauss map, the partial derivatives of $G$, components and determinant of the matrix $\left(g_{i j}\right)$ are obtained as $G_{u}=-\tau N, G_{u u}=\kappa \tau T-\tau^{\prime} N-\tau^{2} B, G_{v}=G_{u v}=G_{v v}=0,\left\langle x_{u}, x_{u}\right\rangle=(\sin \theta-\nu \eta)^{2}+$ $\cos ^{2} \theta,\left\langle x_{u}, x_{v}\right\rangle=\left\langle x_{v}, x_{u}\right\rangle=\cos \theta,\left\langle x_{v}, x_{v}\right\rangle=1, g=(\sin \theta-\nu \eta)^{2}$ respectively. Then, the Laplacian of Gauss map is

$$
\Delta G=-\kappa \tau \zeta T+\left[-\tau \zeta^{3}\left(\zeta^{-1}\right)_{u}+\tau^{\prime} \zeta^{2}-\tau \cos \theta \zeta \zeta_{v}\right] N+\tau^{2} \zeta^{2} B
$$

If $\tau=0$, then the surface is a plane and $\Delta G=0$ holds. So, we consider the second case $\sin \theta=0$ for the Laplacian of Gauss map. For this case, we have $\cos \theta=1$ and from (5.1) it follows $\Delta G=-\frac{1}{v^{2} \eta^{2}}\left(\kappa \tau T-\tau^{\prime} N-\tau^{2} B\right)$ which gives that $\Delta G \neq f G$ since we assume $\tau \neq 0$. In this case, the dot-surface $M$ does not have 1-type Gauss map. Therefore, we have the following theorem:

Theorem 11. A dot-surface M has pointwise 1-type Gauss map of first kind if and only if $M$ is a plane.

For the Laplacian of the position vector $x$ of $M$, we calculate the partial derivatives as follows $x_{u u}=\kappa N+v\left(-\eta^{\prime} r-\eta^{2} q_{o}\right), \quad x_{u v}=-\eta r, x_{v v}=0$ and investigate the following cases:

Case 1: Let $\tau=0$ and $\sin \theta \neq 0$. Then, the Laplacian of the position vector $x$ of the surface $M$ is obtained as

$$
\begin{aligned}
\Delta x=-\zeta\{ & -\zeta^{2}\left(\zeta^{-1}\right)_{u}-\zeta_{v} \cos \theta \\
& +\left[-v \eta^{2} \zeta-(\zeta \cos \theta)_{u}+\left(\zeta\left(\zeta^{-2}+\cos ^{2} \theta\right)\right)_{v}\right] \cos \theta \\
& \left.+\left[\nu \eta \zeta^{2}\left(\zeta^{-1}\right)_{u}-v \eta^{\prime} \zeta+2 \eta \zeta \cos \theta-v \eta \zeta_{v} \cos \theta\right] \sin \theta\right\} T \\
-\zeta\{ & \kappa \zeta+\left[-v \eta^{2} \zeta-(\zeta \cos \theta)_{u}+\left(\zeta\left(\zeta^{-2}+\cos ^{2} \theta\right)\right)_{v}\right] \sin \theta \\
& \left.-\left[\nu \eta \zeta^{2}\left(\zeta^{-1}\right)_{u}-v \eta^{\prime} \zeta+2 \eta \zeta \cos \theta-v \eta \zeta_{v} \cos \theta\right] \cos \theta\right\} N .
\end{aligned}
$$

When $\tau=0$, i.e., the base curve is planar, then we have that the dot-surface is a plane. Then, the equality given in (5.2) is always equal to zero, $\Delta x=0$, in Case 1. Thus, we have the following corollary:

Corollary 1. A dot-surface $M$ with a planar base curve satisfy the equations

$$
\left\{\begin{array}{l}
-\zeta^{2}\left(\zeta^{-1}\right)_{u}-\zeta_{v} \cos \theta+y_{1} \cos \theta+y_{2} \sin \theta=0 \\
\kappa \zeta+y_{1} \sin \theta-y_{2} \cos \theta=0
\end{array}\right.
$$



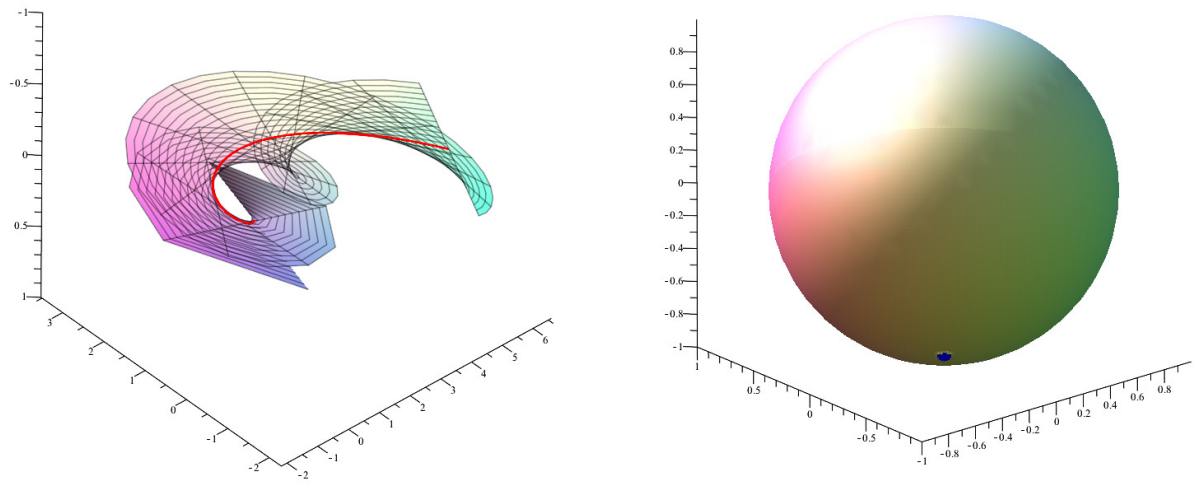

FIGURE 5. $M_{5}$ with base curve $\alpha$ (red) and Gauss map (blue).

where

$$
\begin{aligned}
& y_{1}=-v \eta^{2} \zeta-(\cos \theta \zeta)_{u}+\left(\zeta\left(\zeta^{-2}+\cos ^{2} \theta\right)\right)_{v}, \\
& y_{2}=v \eta \zeta^{2}\left(\zeta^{-1}\right)_{u}-v \eta^{\prime} \zeta+2 \eta \cos \theta \zeta-v \eta \cos \theta \zeta_{v} .
\end{aligned}
$$

Case 2: Let $\tau \neq 0$ and $\sin \theta=0$. Then, the Laplacian of the position vector $x$ of $M$ is given by $\Delta x=\frac{2}{v^{3} \eta^{2}} T+\frac{\theta^{\prime}}{v^{2} \eta^{2}} N$. Therefore, in this case, we get $\Delta x \neq 0$.

From the above cases, the following theorem is obtained:

Theorem 12. A dot-surface $M$ has harmonic type position vector (or, equivalently minimal) if and only if its base curve is planar.

Example 5. Let consider the plane curve $\alpha$ given by the parametrization

$$
\alpha(u)=\left(\left(\frac{u}{\sqrt{2}}+1\right) \cos \left(\ln \left(\frac{u}{\sqrt{2}}+1\right)\right),\left(\frac{u}{\sqrt{2}}+1\right) \sin \left(\ln \left(\frac{u}{\sqrt{2}}+1\right)\right), 0\right) .
$$

Then, by choosing $\theta(u)=u / \sqrt{2}$, a $d o t$-surface $M_{5}$ can be given by the parametrization

$$
x(u, v)=\alpha(u)+v\left(\cos \left(\frac{u}{\sqrt{2}}\right) T(u)+\sin \left(\frac{u}{\sqrt{2}}\right) N(u)\right)
$$

where $T(u)$ and $N(u)$ are tangent vector and principal vector, respectively. The Gauss map of $d o t$-surface $M_{5}$ is computed as $G(u, v)=(0,0,-1)$ and finally, we obtain that $\Delta G=0$ which indicates that the dot-surface $M_{5}$ has pointwise 1-type Gauss map of the first kind. Furthermore, since the base curve $\alpha$ is planar, the surface $M_{5}$ is a plane. Then, it is minimal and has harmonic type position vector. The graph of the dot-surface $M_{5}$ is given in Figure 5. 


\section{REFERENCES}

[1] C. Baikoussis, "Ruled submanifolds with finite type Gauss map." J. Geom., vol. 49, pp. 42-45, 1994, doi: 10.1007/BF01228047.

[2] C. Baikoussis, B.-Y. Chen, and L. Verstraelen, Surfaces with Finite Type Gauss Map. Singapore: Geometry and Topology of Submanifolds, vol. IV, World Scientific, 1992. doi: $10.1142 / 9789814537346$.

[3] C. Baikoussis, B.-Y. Chen, and L. Verstraelen, "Ruled surfaces and tubes with finite type Gauss map.” Tokyo J. Math., vol. 16, pp. 341-348, 1993, doi: 10.3836/tjm/1270128488.

[4] B.-Y. Chen, Total mean curvature and submanifolds of finite type. New Jersey: World Scientific Publ., 1984.

[5] B.-Y. Chen, Finite type submanifolds and generalizations. Dipartimento di Matematica.: Universita degli Studi di Roma "La Sapienza", 1985.

[6] B.-Y. Chen, M. Choi, and Y. H. Kim, "Surfaces of revolution with pointwise 1-type Gauss map." J. Korean Math. Soc., vol. 42, no. 3, pp. 447-455, 2005, doi: 10.4134/JKMS.2005.42.3.447.

[7] B.-Y. Chen and P. Piccini, "Submanifolds with finite type Gauss map." Bull. Austral. Math. Soc., vol. 35, no. 2, pp. 161-186, 1987, doi: 10.1017/S0004972700013162.

[8] M. P. do Carmo, Differential Geometry of Curves and Surfaces. New Jersey: Prentice-Hall, 1976.

[9] M. Emmer, Imagine Math Between Culture and Mathematics. New Jersey: Springer, 2012 ed., 2012.

[10] A. Karger and J. Novak, Space Kinematics and Lie Groups. Prague, Czechoslovakia: STNL Publishers of Technical Lit., 1978.

[11] O. Kaya, T. Kahraman, and M. Önder, "Osculating-type Ruled Surfaces in the Euclidean 3-space." arXiv:2006.06420 [math.DG].

[12] O. Kaya and M. Önder, "Generalized Normal Ruled Surfaces of a Curve in the Euclidean 3-space." arXiv:2006.00242 [math.DG].

[13] D.-S. Kim, "Surfaces with Pointwise 1-Type Gauss Map." J. Korean Soc. Math. Educ. Ser. B: Pure Appl. Math., vol. 18, no. 4, pp. 369-377, 2011, doi: 10.7468/jksmeb.2011.18.4.369.

[14] Y. H. Kim and D. W. Yoon, "Ruled surfaces with pointwise 1-type Gauss map." J. Geom. Phys., vol. 34, pp. 191-205, 2000, doi: 10.1016/S0393-0440(99)00063-7.

[15] M. Önder and T. Kahraman, "On rectifying ruled surfaces." Kuwait J. Sci., vol. 47, no. 4, pp. 1-11, 2020.

[16] M. A. Soliman, H. N. Abd-Ellah, S. A. Hassan, and S. Q. Saleh, "Darboux Ruled Surfaces with Pointwise 1-type Gauss map.” Sohag J. Sci., vol. 2, no. 1, pp. 1-8, 2017, doi: 10.18576/sjs/020101.

Authors' addresses

O. Kaya

(Corresponding author) Manisa Celal Bayar University, Department of Mathematics, 45140 Manisa, Turkey

E-mail address: onur.kayaecbu.edu.tr

\section{M. Önder}

Delibekirli Village, Tepe Street, Kırıkhan, 31440 Hatay, Turkey

E-mail address: mehmetonder197999@gmail.com 